UDC: $517.518 .28: 517.518 .18$

44 (LXX) 2 (2020), 119-129

DOI: $10.37560 /$ matbil2020119k

\title{
SOME NEW INTEGRAL INEQUALITIES FOR $\rho$-CONVEX FUNCTIONS
}

\author{
ARTION KASHURI ${ }^{1}$, MUHAMMAD AAMIR ALI ${ }^{2}$, AND MUJAHID ABBAS ${ }^{3}$
}

\begin{abstract}
The aim of this paper is to generalize the results in 10 using the class of $\rho$-convex functions. Some special cases are deduced from main results. Applying our ideas and techniques, new interesting inequalities can be obtained in a similar way for different class of functions and operators.
\end{abstract}

\section{Introduction and Preliminaries}

The class of convex functions is well known in the literature and is defined in the following way:

Definition 1.1. Let $I$ be an interval in $\Re$. A function $f: I \rightarrow \Re$, is said to be convex on $I$, if the inequality

$$
f(\gamma u+(1-\gamma) v) \leq \gamma f(u)+(1-\gamma) f(v)
$$

holds for all $u, v \in I$ and $\gamma \in[0,1]$. Also, we say that $f$ is concave, if the inequality in (1.1) holds in the reverse direction.

Theory of convex functions and their variant forms is used to study a wide class of problems that arise in various branches of pure and applied sciences. This theory provides us with a natural, unified and general framework for solving a wide class of optimization problems.

The following inequality, named Hermite-Hadamard inequality, is one of the most famous inequalities in the literature for convex functions.

Theorem 1. Let $f: I \subseteq \Re \rightarrow \Re$ be a convex function and $u, v \in I$ with $u<v$. Then the following inequality holds:

$$
f\left(\frac{u+v}{2}\right) \leq \frac{1}{v-u} \int_{u}^{v} f(x) d x \leq \frac{f(u)+f(v)}{2} .
$$

This inequality (1.2) is also known as trapezium inequality.

2010 Mathematics Subject Classification. Primary: 26D07 Secondary: 26A33, 26A51, 90C23, 26D10, 26D15.

Key words and phrases. Hermite-Hadamard integral inequality; $\rho$-convex function; arithmetic-geometric means inequality. 
The trapezium inequality has remained an area of great interest due to its wide applications in the field of mathematical analysis. Authors of recent decades have studied (1.2) in the premises of newly invented definitions due to motivation of convex function. For applications, generalizations and other aspects of convex functions, Hermite-Hadamard's inequality and their variant forms, see the references [1]- 10].

The motivation of this paper is not only the generalization of the known results by $\mathrm{Wu}$ et al. in 10 but using our new ideas and techniques we can find some new interesting results for different operators and classes of functions as well. About their applications this will be investigate in our next paper.

Now, let us recall the following useful definitions.

Definition 1.2. A set $\mathcal{S} \subseteq \Re$ is said to be convex, if $(1-\gamma) u+\gamma v \in \mathcal{S}$ for every $u, v \in \mathcal{S}$ and $\gamma \in[0,1]$.

Recently, Wu et al. [10] introduced the following new classes of $\rho$-convex sets and $\rho$-convex functions.

Definition 1.3. A set $\mathcal{S} \subseteq \Re$ is said to be $\rho$-convex with respect to strictly monotonic continuous function $\rho$, if

$$
\mathcal{M}_{[\rho]}(u, v):=\rho^{-1}((1-\gamma) \rho(u)+\gamma \rho(v)) \in \mathcal{S}, \quad \forall u, v \in \mathcal{S}, \gamma \in[0,1] .
$$

Definition 1.4. A function $\mathcal{S} \subseteq \Re$ is said to be $\rho$-convex with respect to strictly monotonic continuous function $\rho$, if

$$
f\left(\mathcal{M}_{[\rho]}(u, v)\right) \leq(1-\gamma) f(u)+\gamma f(v), \quad \forall u, v \in \mathcal{S}, \gamma \in[0,1] .
$$

Note that the function $f$ is called strictly $\rho$-convex on $\mathcal{S}$ if the above inequality is true as a strict inequality for each distinct $u$ and $v$ in $\mathcal{S}$ and for each $\gamma \in(0,1)$. The function $f$ is called $\rho$-concave (strictly $\rho$-concave) on $\mathcal{S}$, if $(-f)$ is $\rho$-convex (strictly $\rho$-convex) on $\mathcal{S}$.

The main objective of this article is to generalize the results by $\mathrm{Wu}$ et al. in [10] using the class of $\rho$-convex functions. We shall also discuss some of its special cases. At the end, a briefly conclusion will be given.

\section{Main Results}

Theorem 2. Suppose that $f: I \rightarrow \Re$ is an integrable $\rho$-convex function with respect to the function $\rho$. Then for $\alpha>0$, the following double integral inequalities hold:

$$
\begin{gathered}
f\left(\rho^{-1}\left(\frac{\rho(u)+\rho(v)}{2}\right)\right) \leq \frac{\alpha}{2(\rho(v)-\rho(u))^{\alpha}} \\
\times\left[\int_{u}^{v}(\rho(x)-\rho(u))^{\alpha-1} f(x) \rho^{\prime}(x) d x+\int_{u}^{v}(\rho(v)-\rho(x))^{\alpha-1} f(x) \rho^{\prime}(x) d x\right] \\
\leq \frac{f(u)+f(v)}{2}
\end{gathered}
$$


Proof. Since $f$ is a $\rho$-convex function, we have

$$
f\left(\rho^{-1}\left(\frac{\rho(u)+\rho(v)}{2}\right)\right) \leq \frac{f(u)+f(v)}{2} .
$$

Substituting $x=\rho^{-1}((1-\gamma) \rho(u)+\gamma \rho(v))$ and $y=\rho^{-1}(\gamma \rho(u)+(1-\gamma) \rho(v))$ in (2.2), we get

$$
\begin{gathered}
f\left(\rho^{-1}\left(\frac{\rho(u)+\rho(v)}{2}\right)\right) \\
\leq \frac{f\left(\rho^{-1}((1-\gamma) \rho(u)+\gamma \rho(v))\right)+f\left(\rho^{-1}(\gamma \rho(u)+(1-\gamma) \rho(v))\right)}{2} .
\end{gathered}
$$

Multiplying both sides of $(2.3)$ with $\gamma^{\alpha-1}$ and integrating with respect to $\gamma$ on $[0,1]$, we obtain

$$
\begin{gathered}
\int_{0}^{1} \gamma^{\alpha-1} f\left(\rho^{-1}\left(\frac{\rho(u)+\rho(v)}{2}\right)\right) d \gamma \\
\leq \int_{0}^{1} \gamma^{\alpha-1}\left[\frac{f\left(\rho^{-1}((1-\gamma) \rho(u)+t \rho(v))\right)+f\left(\rho^{-1}(t \rho(u)+(1-\gamma) \rho(v))\right)}{2}\right] d \gamma .
\end{gathered}
$$

Hence

$$
\begin{gathered}
f\left(\rho^{-1}\left(\frac{\rho(u)+\rho(v)}{2}\right)\right) \leq \frac{\alpha}{2(\rho(v)-\rho(u))^{\alpha}} \\
\times\left[\int_{u}^{v}(\rho(u)-\rho(u))^{\alpha-1} f(u) \rho^{\prime}(u) d x+\int_{u}^{v}(\rho(v)-\rho(u))^{\alpha-1} f(u) \rho^{\prime}(u) d x\right] .
\end{gathered}
$$

So, the left-hand side of 2.1 is proved. Similarly, since $f$ is a $\rho$-convex function, we have

$$
f\left(\rho^{-1}((1-\gamma) \rho(u)+\gamma \rho(v))\right) \leq(1-\gamma) f(u)+\gamma f(v)
$$

and

$$
f\left(\rho^{-1}(\gamma \rho(u)+(1-\gamma) \rho(v))\right) \leq \gamma f(u)+(1-\gamma) f(v) .
$$

Multiplying both sides of 2.4 and 2.5 with $\gamma^{\alpha-1}$ and integrating with respect to $\gamma$ on $[0,1]$, we get

$$
\begin{gathered}
\int_{0}^{1} \gamma^{\alpha-1}\left[f\left(\rho^{-1}((1-\gamma) \rho(u)+\gamma \rho(v))\right)+f\left(\rho^{-1}(\gamma \rho(u)+(1-\gamma) \rho(v))\right)\right] d \gamma \\
\leq \int_{0}^{1} \gamma^{\alpha-1}[f(u)+f(v)] d \gamma
\end{gathered}
$$

Hence

$$
\begin{gathered}
\frac{\alpha}{2(\rho(v)-\rho(u))^{\alpha}} \\
\times\left[\int_{u}^{v}(\rho(u)-\rho(u))^{\alpha-1} f(u) \rho^{\prime}(u) d x+\int_{u}^{v}(\rho(v)-\rho(u))^{\alpha-1} f(u) \rho^{\prime}(u) d x\right] \\
\leq \frac{f(u)+f(v)}{2} .
\end{gathered}
$$

The proof of Theorem 2 is completed.

Corollary 2.1. Taking $\alpha=1$ in Theorem 2, we get ([10], Theorem 3.1). 
Theorem 3. Suppose that $f: I \rightarrow \Re$ is an integrable $\rho$-convex function with respect to the function $\rho$. Then for $\alpha>0$, the following double integral inequalities hold:

$$
\begin{gathered}
\frac{2 f(u)}{(\rho(v)-\rho(u))^{\alpha+1}} \int_{u}^{v}(\rho(x)-\rho(u))^{\alpha-1}(\rho(v)-\rho(x)) f(x) \rho^{\prime}(x) d x \\
+\frac{2 f(v)}{(\rho(v)-\rho(u))^{\alpha+1}} \int_{u}^{v}(\rho(x)-\rho(u))^{\alpha-1} f(x) \rho^{\prime}(x) d x \\
\leq \frac{1}{(\rho(v)-\rho(u))^{\alpha}} \int_{u}^{v}(\rho(x)-\rho(u))^{\alpha-1} f^{2}(x) \rho^{\prime}(x) d x \\
+\frac{2 f^{2}(u)+\alpha(\alpha+1) f^{2}(v)+2 \alpha f(u) f(v)}{\alpha(\alpha+1)(\alpha+2)} \\
\leq \frac{4 f^{2}(u)+2 \alpha(\alpha+1) f^{2}(v)+4 \alpha f(u) f(v)}{\alpha(\alpha+1)(\alpha+2)} .
\end{gathered}
$$

Proof. Using the arithmetic-geometric means inequality, we have

$$
\begin{gathered}
2 f\left(\rho^{-1}((1-\gamma) \rho(u)+\gamma \rho(v))\right)((1-\gamma) f(u)+\gamma f(v)) \\
\leq\left[f\left(\rho^{-1}((1-\gamma) \rho(u)+\gamma \rho(v))\right)\right]^{2}+[(1-\gamma) f(u)+\gamma f(v)]^{2} \\
=\left[f\left(\rho^{-1}((1-\gamma) \rho(u)+\gamma \rho(v))\right)\right]^{2}+(1-\gamma)^{2} f^{2}(u)+\gamma^{2} f^{2}(v)+2 \gamma(1-\gamma) f(u) f(v) .
\end{gathered}
$$

Multiplying both sides of 2.7 with $\gamma^{\alpha-1}$ and integrating with respect to $\gamma$ on $[0,1]$, we get

$$
\begin{gathered}
2 f(u) \int_{0}^{1} \gamma^{\alpha-1}(1-\gamma)\left[f\left(\rho^{-1}((1-\gamma) \rho(u)+\gamma \rho(v))\right)\right] d \gamma \\
+2 f(v) \int_{0}^{1} \gamma^{\alpha-1} \gamma\left[f\left(\rho^{-1}((1-\gamma) \rho(u)+\gamma \rho(v))\right)\right] d \gamma \\
\leq \int_{0}^{1} \gamma^{\alpha-1}\left[f\left(\rho^{-1}((1-\gamma) \rho(u)+\gamma \rho(v))\right)\right]^{2} d \gamma+f^{2}(u) \int_{0}^{1} \gamma^{\alpha-1}(1-\gamma)^{2} d \gamma \\
+f^{2}(v) \int_{0}^{1} \gamma^{\alpha-1} \gamma^{2} d \gamma+2 f(u) f(v) \int_{0}^{1} \gamma^{\alpha-1} \gamma(1-\gamma) d \gamma
\end{gathered}
$$

Substituting $x=\rho^{-1}((1-\gamma) \rho(u)+\gamma \rho(v))$ in $(2.8)$, we get

$$
\begin{gathered}
\frac{2 f(u)}{(\rho(v)-\rho(u))^{\alpha+1}} \int_{u}^{v}(\rho(u)-\rho(u))^{\alpha-1}(\rho(v)-\rho(u)) f(u) \rho^{\prime}(u) d x \\
+\frac{2 f(v)}{(\rho(v)-\rho(u))^{\alpha+1}} \int_{u}^{v}(\rho(u)-\rho(u))^{\alpha-1} f(u) \rho^{\prime}(u) d x \\
\leq \frac{1}{(\rho(v)-\rho(u))^{\alpha}} \int_{u}^{v}(\rho(u)-\rho(u))^{\alpha-1} f^{2}(u) \rho^{\prime}(u) d x \\
+\frac{2 f^{2}(u)+\alpha(\alpha+1) f^{2}(v)+2 \alpha f(u) f(v)}{\alpha(\alpha+1)(\alpha+2)}
\end{gathered}
$$


So the left-hand side of 2.6$]$ is proved. On the other hand, since $f$ is a $\rho$-convex function, we have

$$
f\left(\rho^{-1}((1-\gamma) \rho(u)+\gamma \rho(v))\right) \leq(1-\gamma) f(u)+\gamma f(v) .
$$

Hence

$$
\begin{gathered}
\frac{1}{(\rho(v)-\rho(u))^{\alpha}} \int_{u}^{v}(\rho(u)-\rho(u))^{\alpha-1} f^{2}(u) \rho^{\prime}(u) d x \\
=\int_{0}^{1} \gamma^{\alpha-1}\left[f\left(\rho^{-1}((1-\gamma) \rho(u)+\gamma \rho(v))\right)\right]^{2} d \gamma \\
\leq \int_{0}^{1} \gamma^{\alpha-1}[(1-\gamma) f(u)+\gamma f(v)]^{2} d \gamma \\
=\frac{2 f^{2}(u)+\alpha(\alpha+1) f^{2}(v)+2 \alpha f(u) f(v)}{\alpha(\alpha+1)(\alpha+2)} .
\end{gathered}
$$

Combining (2.9) and (2.11) we obtain the right-hand side of (2.6). The proof of Theorem 3 is completed.

Corollary 3.1. Taking $\alpha=1$ in Theorem 3, we get (10, Theorem 3.2).

Theorem 4. Suppose that $f, h: I \rightarrow \Re^{+}$are two integrable $\rho$-convex functions with respect to the function $\rho$. Then for $\alpha>0$, the following double integral inequalities hold:

$$
\begin{aligned}
& \frac{1}{(\rho(v)-\rho(u))^{\alpha}} \int_{u}^{v}(\rho(x)-\rho(u))^{\alpha-1} f(x) h(x) \rho^{\prime}(x) d x \\
& \quad \leq \frac{2 f(u) h(u)}{\alpha(\alpha+1)(\alpha+2)}+\frac{f(v) h(v)}{\alpha+2}+\frac{N(u, v)}{(\alpha+1)(\alpha+2)} \\
& \quad \leq \frac{F(u)}{\alpha(\alpha+1)(\alpha+2)}+\frac{F(v)}{2(\alpha+2)}+\frac{P(u, v)}{(\alpha+1)(\alpha+2)}
\end{aligned}
$$

where

$$
N(u, v)=f(u) h(v)+f(v) h(u), \quad P(u, v)=f(u) f(v)+h(u) h(v)
$$

and

$$
F(u)=f^{2}(u)+h^{2}(u), \quad F(v)=f^{2}(v)+h^{2}(v) .
$$

Proof. Since $f$ and $h$ are two integrable $\rho$-convex functions, we have

$$
\begin{gathered}
f\left(\rho^{-1}((1-\gamma) \rho(u)+\gamma \rho(v))\right) h\left(\rho^{-1}((1-\gamma) \rho(u)+\gamma \rho(v))\right) \\
\leq[(1-\gamma) f(u)+\gamma f(v)][(1-\gamma) h(u)+\gamma h(v)] .
\end{gathered}
$$

Multiplying both sides of 2.15) with $\gamma^{\alpha-1}$ and integrating with respect to $\gamma$ on $[0,1]$, we obtain

$$
\begin{gathered}
\int_{0}^{1} \gamma^{\alpha-1} f\left(\rho^{-1}((1-\gamma) \rho(u)+\gamma \rho(v))\right) h\left(\rho^{-1}((1-\gamma) \rho(u)+\gamma \rho(v))\right) d \gamma \\
\leq \int_{0}^{1} \gamma^{\alpha-1}[(1-\gamma) f(u)+\gamma f(v)][(1-\gamma) h(u)+\gamma h(v)] d \gamma
\end{gathered}
$$




$$
\begin{gathered}
=f(u) h(u) \int_{0}^{1} \gamma^{\alpha-1}(1-\gamma)^{2} d \gamma+N(u, v) \int_{0}^{1} \gamma^{\alpha-1} \gamma(1-\gamma) d \gamma \\
+f(v) h(v) \int_{0}^{1} \gamma^{\alpha-1} \gamma^{2} d \gamma .
\end{gathered}
$$

Substituting $x=\rho^{-1}((1-\gamma) \rho(u)+\gamma \rho(v))$ in 2.16$)$, we obtain the left-hand side of 2.12). For the right-hand side inequality, using the arithmetic-geometric means inequality in 2.16, gives

$$
\begin{gathered}
\int_{0}^{1} \gamma^{\alpha-1}[(1-\gamma) f(u)+\gamma f(v)][(1-\gamma) h(u)+\gamma h(v)] d \gamma \\
\leq \int_{0}^{1} \gamma^{\alpha-1}\left[\frac{[(1-\gamma) f(u)+\gamma f(v)]^{2}+[(1-\gamma) h(u)+\gamma h(v)]^{2}}{2}\right] d \gamma \\
=\frac{1}{2} \int_{0}^{1}\left[F(u) \gamma^{\alpha-1}(1-\gamma)^{2}+F(v) \gamma^{\alpha-1} \gamma^{2}+2 P(u, v) \gamma^{\alpha-1} \gamma(1-\gamma)\right] d \gamma \\
=\frac{F(u)}{\alpha(\alpha+1)(\alpha+2)}+\frac{F(v)}{2(\alpha+2)}+\frac{P(u, v)}{(\alpha+1)(\alpha+2)} .
\end{gathered}
$$

The proof of Theorem 4 is completed.

Corollary 4.1. Taking $\alpha=1$ in Theorem 4, we get ([10], Theorem 3.5).

Theorem 5. Suppose that $f, h: I \rightarrow \Re^{+}$are two similarly ordered integrable $\rho$-convex functions with respect to the function $\rho$. Then for $\alpha>0$, the following integral inequality holds:

$$
\frac{1}{(\rho(v)-\rho(u))^{\alpha}} \int_{u}^{v}(\rho(x)-\rho(u))^{\alpha-1} f(x) h(x) \rho^{\prime}(x) d x \leq \frac{M(\alpha, u, v)}{\alpha(\alpha+1)},
$$

where

$$
M(\alpha, u, v)=f(u) h(u)+\alpha f(v) h(v) .
$$

Proof. Since $f$ and $h$ are two similarly ordered integrable $\rho$-convex functions with respect to the function $\rho$, we have

$$
\begin{gathered}
f\left(\rho^{-1}((1-\gamma) \rho(u)+\gamma \rho(v))\right) h\left(\rho^{-1}((1-\gamma) \rho(u)+\gamma \rho(v))\right) \\
\leq[(1-\gamma) f(u)+\gamma f(v)][(1-\gamma) h(u)+\gamma h(v)] \\
=(1-\gamma) f(u) h(u)+\gamma f(v) h(v)-\gamma(1-\gamma)[(f(u)-f(v))(h(u)-h(v))] \\
\leq(1-\gamma) f(u) h(u)+\gamma f(v) h(v),
\end{gathered}
$$

where $(f(u)-f(v))(h(u)-h(v)) \geq 0$. Multiplying both sides of 2.19) with $\gamma^{\alpha-1}$ and integrating with respect to $\gamma$ on $[0,1]$, we get

$$
\begin{gathered}
\int_{0}^{1} \gamma^{\alpha-1} f\left(\rho^{-1}((1-\gamma) \rho(u)+\gamma \rho(v))\right) h\left(\rho^{-1}((1-\gamma) \rho(u)+\gamma \rho(v))\right) d \gamma \\
\leq \int_{0}^{1} \gamma^{\alpha-1}[(1-\gamma) f(u) h(u)+\gamma f(v) h(v)] d \gamma
\end{gathered}
$$

Substituting $x=\rho^{-1}((1-\gamma) \rho(u)+\gamma \rho(v))$ in 2.20), we obtain 2.17). The proof of Theorem 5 is completed. 
Corollary 5.1. Taking $\alpha=1$ in Theorem [5, we get ([10], Theorem 3.6).

Theorem 6. Suppose that $f, h: I \rightarrow \Re^{+}$are two similarly ordered integrable $\rho-$ convex functions with respect to the function $\rho$. Then for $\alpha>0$, the following integral inequality holds:

$$
\frac{1}{(\rho(v)-\rho(u))^{\alpha}} \int_{u}^{v}(\rho(v)-\rho(x))^{\alpha-1} f(x) h(x) \rho^{\prime}(x) d x \leq \frac{R(\alpha, u, v)}{\alpha(\alpha+1)},
$$

where

$$
R(\alpha, u, v)=\alpha f(u) h(u)+f(v) h(v) .
$$

Proof. The proof is similar as Theorem 5, so we omit it.

Corollary 6.1. Taking $\alpha=1$ in Theorem 6 , we get the following integral inequality:

$$
\frac{1}{\rho(v)-\rho(u)} \int_{u}^{v} f(x) h(x) \rho^{\prime}(x) d x \leq \frac{f(u) h(u)+f(v) h(v)}{2} .
$$

Theorem 7. Suppose that $f, h: I \rightarrow \Re^{+}$are two integrable $\rho$-convex functions with respect to the function $\rho$. Then for $\alpha>0$, the following integral inequality holds:

$$
\begin{gathered}
2 f\left(\rho^{-1}\left(\frac{\rho(u)+\rho(v)}{2}\right)\right) h\left(\rho^{-1}\left(\frac{\rho(u)+\rho(v)}{2}\right)\right)-\frac{\alpha}{2(\rho(v)-\rho(u))^{\alpha}} \\
\times\left[\int_{u}^{v}(\rho(x)-\rho(u))^{\alpha-1} f(x) h(x) \rho^{\prime}(x) d x+\int_{u}^{v}(\rho(v)-\rho(x))^{\alpha-1} f(x) h(x) \rho^{\prime}(x) d x\right] \\
\leq \frac{\alpha}{(\alpha+1)(\alpha+2)} M(1, u, v)+\left[\frac{2+\alpha(\alpha+1)}{2 \alpha(\alpha+1)(\alpha+2)}\right] N(u, v),
\end{gathered}
$$

where $N(u, v)$ is defined from (2.13) and $M(1, u, v)$ is defined from (2.18) for value $\alpha=1$.

Proof. Since $f$ and $h$ are two integrable $\rho$-convex functions with respect to the function $\rho$, by the same way as in the proof of Theorem 2.1. we have

$$
\begin{gathered}
f\left(\rho^{-1}\left(\frac{\rho(u)+\rho(v)}{2}\right)\right) h\left(\rho^{-1}\left(\frac{\rho(u)+\rho(v)}{2}\right)\right) \\
\leq \frac{1}{4}\left[f\left(\rho^{-1}((1-\gamma) \rho(u)+\gamma \rho(v))\right)+f\left(\rho^{-1}(\gamma \rho(u)+(1-\gamma) \rho(v))\right)\right] \\
\times\left[h\left(\rho^{-1}((1-\gamma) \rho(u)+\gamma \rho(v))\right)+h\left(\rho^{-1}(\gamma \rho(u)+(1-\gamma) \rho(v))\right)\right] \\
\leq \frac{1}{4}\left\{f\left(\rho^{-1}((1-\gamma) \rho(u)+\gamma \rho(v))\right) h\left(\rho^{-1}((1-\gamma) \rho(u)+\gamma \rho(v))\right)\right. \\
+f\left(\rho^{-1}(t \rho(u)+(1-\gamma) \rho(v))\right) h\left(\rho^{-1}(t \rho(u)+(1-\gamma) \rho(v))\right) \\
+[(1-\gamma) f(u)+\gamma f(v)][t h(u)+(1-\gamma) h(v)] \\
+[\gamma f(u)+(1-\gamma) f(v)][(1-\gamma) h(u)+\gamma h(v)]\} .
\end{gathered}
$$


Multiplying both sides of 2.25 with $\gamma^{\alpha-1}$ and integrating with respect to $\gamma$ on $[0,1]$, we obtain

$$
\begin{gathered}
f\left(\rho^{-1}\left(\frac{\rho(u)+\rho(v)}{2}\right)\right) h\left(\rho^{-1}\left(\frac{\rho(u)+\rho(v)}{2}\right)\right) \\
\leq \frac{\alpha}{4}\left\{\int_{0}^{1} \gamma^{\alpha-1} f\left(\rho^{-1}((1-\gamma) \rho(u)+\gamma \rho(v))\right) h\left(\rho^{-1}((1-\gamma) \rho(u)+\gamma \rho(v))\right) d \gamma\right. \\
+\int_{0}^{1} \gamma^{\alpha-1} f\left(\rho^{-1}(\gamma \rho(u)+(1-\gamma) \rho(v))\right) h\left(\rho^{-1}(\gamma \rho(u)+(1-\gamma) \rho(v))\right) d \gamma \\
\left.+2 M(1, u, v) \int_{0}^{1} \gamma^{\alpha-1} \gamma(1-\gamma) d t+N(u, v) \int_{0}^{1} \gamma^{\alpha-1}\left(\gamma^{2}+(1-\gamma)^{2}\right) d \gamma\right\} .
\end{gathered}
$$

Substituting $x=\rho^{-1}((1-\gamma) \rho(u)+\gamma \rho(v))$ and $y=\rho^{-1}(\gamma \rho(u)+(1-\gamma) \rho(v))$ in 2.26, we obtain 2.24. The proof of Theorem 7 is completed.

Corollary 7.1. Taking $\alpha=1$ in Theorem 7, we get ([10, Theorem 3.7).

Theorem 8. Suppose that $f, h: I \rightarrow \Re^{+}$are two integrable $\rho$-convex functions with respect to the function $\rho$. Then for $\alpha>0$, the following integral inequality holds:

$$
\begin{gathered}
f\left(\rho^{-1}\left(\frac{\rho(u)+\rho(v)}{2}\right)\right) h\left(\rho^{-1}\left(\frac{\rho(u)+\rho(v)}{2}\right)\right) \\
\leq\left[\frac{1}{4}+\frac{\alpha}{(\alpha+1)(\alpha+2)}\right] M(1, u, v)+\left[\frac{2+\alpha(\alpha+1)}{4 \alpha(\alpha+1)(\alpha+2)}\right] N(u, v),
\end{gathered}
$$

where $N(u, v)$ is defined from (2.13) and $M(1, u, v)$ is defined from (2.18) for value $\alpha=1$.

Proof. From Theorems 5, 6 and Theorem 7, we get the desired inequality (2.27).

Corollary 8.1. Taking $\alpha=1$ in Theorem 8, we get the following integral inequality:

$$
f\left(\rho^{-1}\left(\frac{\rho(u)+\rho(v)}{2}\right)\right) h\left(\rho^{-1}\left(\frac{\rho(u)+\rho(v)}{2}\right)\right) \leq \frac{5 M(1, u, v)+2 N(u, v)}{12} .
$$

Theorem 9. Suppose that $f, h: I \rightarrow \Re^{+}$are two integrable $\rho$-convex functions with respect to the function $\rho$. Then for $\alpha>0$, the following double integral inequalities hold:

$$
\begin{gathered}
\int_{u}^{v} \int_{u}^{v} \int_{0}^{1} t^{\alpha-1}(\rho(x)-\rho(u))^{\alpha-1}(\rho(y)-\rho(u))^{\alpha-1} f\left(\rho^{-1}((1-\gamma) \rho(x)+\gamma \rho(y))\right) \\
\quad \times h\left(\rho^{-1}((1-\gamma) \rho(x)+\gamma \rho(y))\right) \rho^{\prime}(x) \rho^{\prime}(y) d \gamma d y d x \\
\leq \frac{(2+\alpha(\alpha+1))}{\alpha^{2}(\alpha+1)(\alpha+2)}(\rho(v)-\rho(u))^{2 \alpha} D(\alpha, u, v)+\frac{1}{(\alpha+1)(\alpha+2)}
\end{gathered}
$$




$$
\begin{gathered}
\times\left\{\int_{u}^{v}(\rho(y)-\rho(u))^{\alpha-1} h(y) \rho^{\prime}(y) d y \int_{u}^{v}(\rho(x)-\rho(u))^{\alpha-1} f(x) \rho^{\prime}(x) d x\right. \\
\left.+\int_{u}^{v}(\rho(y)-\rho(u))^{\alpha-1} f(y) \rho^{\prime}(y) d y \int_{u}^{v}(\rho(x)-\rho(u))^{\alpha-1} h(x) \rho^{\prime}(x) d x\right\} \\
\leq \frac{(2+\alpha(\alpha+1))}{\alpha^{2}(\alpha+1)(\alpha+2)}(\rho(v)-\rho(u))^{2 \alpha} D(\alpha, u, v) \\
+\frac{(f(u)+f(v))(h(u)+h(v))}{2(\alpha+1)(\alpha+2)}(\rho(v)-\rho(u))^{2 \alpha},
\end{gathered}
$$

where

$$
D(\alpha, u, v)=\frac{2 f(u) h(u)}{\alpha(\alpha+1)(\alpha+2)}+\frac{f(v) h(v)}{\alpha+2}+\frac{N(u, v)}{(\alpha+1)(\alpha+2)}
$$

and $N(u, v)$ is defined from (2.13).

Proof. Since $f$ and $h$ are two integrable $\rho$-convex functions with respect to the function $\rho$, we have

$$
\begin{gathered}
f\left(\rho^{-1}((1-\gamma) \rho(u)+\gamma \rho(v))\right) h\left(\rho^{-1}((1-\gamma) \rho(u)+\gamma \rho(v))\right) \\
\leq[(1-\gamma) f(u)+\gamma f(v)][(1-\gamma) h(u)+\gamma h(v)] \\
=(1-\gamma)^{2} f(u) h(u)+\gamma(1-\gamma)[f(u) h(v)+h(u) f(v)]+\gamma^{2} f(v) h(v) .
\end{gathered}
$$

Multiplying both sides of 2.31 with $\gamma^{\alpha-1}$ and integrating with respect to $\gamma$ on $[0,1]$, we obtain

$$
\begin{gathered}
\int_{0}^{1} \gamma^{\alpha-1} f\left(\rho^{-1}((1-\gamma) \rho(u)+\gamma \rho(v))\right) h\left(\rho^{-1}((1-\gamma) \rho(u)+\gamma \rho(v))\right) d \gamma \\
\leq f(u) h(u) \int_{0}^{1} \gamma^{\alpha-1}(1-\gamma)^{2} d \gamma+f(v) h(v) \int_{0}^{1} \gamma^{\alpha-1} \gamma^{2} d \gamma \\
+[f(u) h(v)+h(u) f(v)] \int_{0}^{1} \gamma^{\alpha-1} \gamma(1-\gamma) d \gamma \\
=\frac{2 f(u) h(u)}{\alpha(\alpha+1)(\alpha+2)}+\frac{f(v) h(v)}{\alpha+2}+\frac{f(u) h(v)+h(u) f(v)}{(\alpha+1)(\alpha+2)}
\end{gathered}
$$

Again, integrating both sides of 2.32 over the plane domain $\{(x, y): x \in[u, v], y \in$ $[u, v]\}$ and then using the left-hand side of Theorem 4 , we deduce the left-hand side of 2.29 .

On the other hand, substituting $x=\rho^{-1}((1-\gamma) \rho(u)+\gamma \rho(v))$ and using again the fact that $f$ and $h$ are two integrable $\rho$-convex functions with respect to the function $\rho$, we get

$$
\begin{gathered}
\int_{u}^{v}(\rho(x)-\rho(u))^{\alpha-1} f(x) \rho^{\prime}(x) d x=(\rho(v)-\rho(u))^{\alpha} \\
\times \int_{0}^{1} f\left(\rho^{-1}((1-\gamma) \rho(u)+\gamma \rho(v))\right) d \gamma \\
\leq \frac{(f(u)+f(v))}{2}(\rho(v)-\rho(u))^{\alpha}
\end{gathered}
$$


Similarly, we have

$$
\begin{aligned}
& \int_{u}^{v}(\rho(y)-\rho(u))^{\alpha-1} f(y) \rho^{\prime}(y) d y \leq \frac{(f(u)+f(v))}{2}(\rho(v)-\rho(u))^{\alpha} . \\
& \int_{u}^{v}(\rho(x)-\rho(u))^{\alpha-1} h(x) \rho^{\prime}(x) d x \leq \frac{(h(u)+h(v))}{2}(\rho(v)-\rho(u))^{\alpha} . \\
& \int_{u}^{v}(\rho(y)-\rho(u))^{\alpha-1} h(y) \rho^{\prime}(y) d y \leq \frac{(h(u)+h(v))}{2}(\rho(v)-\rho(u))^{\alpha} .
\end{aligned}
$$

Combining (2.33)-2.36), we obtain the right-hand side of 2.29 . The proof of Theorem 9 is completed.

Corollary 9.1. Taking $\alpha=1$ in Theorem 9, we get the following double integral inequalities:

$$
\begin{gathered}
\int_{u}^{v} \int_{u}^{v} \int_{0}^{1} f\left(\rho^{-1}((1-\gamma) \rho(x)+\gamma \rho(y))\right) \\
\times h\left(\rho^{-1}((1-\gamma) \rho(x)+\gamma \rho(y))\right) \rho^{\prime}(x) \rho^{\prime}(y) d \gamma d y d x \\
\leq \frac{1}{3}\left\{2(\rho(v)-\rho(u))^{2} U(u, v)+\int_{u}^{v} \int_{u}^{v} f(x) h(y) \rho^{\prime}(x) \rho^{\prime}(y) d y d x\right\} \\
\leq \frac{(\rho(v)-\rho(u))^{2}}{3}\left[2 U(u, v)+\frac{(f(u)+f(v))(h(u)+h(v))}{4}\right]
\end{gathered}
$$

where

$$
U(u, v)=\frac{f(u) h(u)+f(v) h(v)}{3}+\frac{N(u, v)}{6}
$$

\section{Conclusion}

Interested reader can obtain in a similar way new results for different operators such as the $k$-Riemann-Liouville fractional integral, Katugampola fractional integrals, the conformable fractional integral, Hadamard fractional integrals, etc. and they can be applied to obtain several interesting results in convex analysis, special functions, quantum mechanics, related optimization theory, mathematical inequalities using different class of functions. Our ideas and techniques may stimulate further research in different areas of pure and applied sciences.

Conflict of interest. All the Authors declares that they have no conflict of interest with anyone. 


\section{REFERENCES}

[1] M. R. Delavar, M. De La Sen, Some generalizations of Hermite-Hadamard type inequalities, SpringerPlus, 5 (1661), (2016), pp. 9.

[2] T. S. Du, M. U. Awan, A. Kashuri, S. Zhao, Some k-fractional extensions of the trapezium inequalities through generalized relative semi- $(m, h)$-preinvexity, Appl. Anal., 100(3), (2021), 642-662.

[3] T. S. Du, J. G. Liao, Y. J. Li, Properties and integral inequalities of Hadamard-Simpson type for the generalized $(s, m)$-preinvex functions, J. Nonlinear Sci. Appl., 9 (2016), 3112-3126.

[4] A. Kashuri, R. Liko, Some new Hermite-Hadamard type inequalities and their applications, Stud. Sci. Math. Hung., 56(1) (2019), 103-142.

[5] M. V. Mihai, Some Hermite-Hadamard type inequalities via Riemann-Liouville fractional calculus, Tamkang J. Math., 44(4) (2013), 411-416.

[6] O. Omotoyinbo, A. Mogbodemu, Some new Hermite-Hadamard integral inequalities for convex functions, Int. J. Sci. Innovation Tech., 1(1) (2014), 1-12.

[7] M. E. Özdemir, S. S. Dragomir, C. Yildiz, The Hadamard's inequality for convex function via fractional integrals, Acta Math. Sci., 33(5), (2013), 153-164.

[8] S. Rashid, M. A. Noor, K. I. Noor, Fractional exponentially $m$-convex functions and inequalities, Int. J. Anal. Appl., 17(3), (2019), 464-478.

[9] Y. Zhang, T. S. Du, H. Wang, Y. J. Shen, A. Kashuri, Extensions of different type parameterized inequalities for generalized $(m, h)$-preinvex mappings via $k$-fractional integrals, J. Inequal. Appl., 2018(49), (2018), 30 pp.

[10] S. Wu, M. U. Awan, M. A. Noor, K. I. Noor, S. Iftikhar, On a new class of convex functions and integral inequalities, J. Inequal. Appl., 2019(131), (2019), 14 pp.

\footnotetext{
1 University "Ismail Qemali", Faculty of Technical Science, Department of Mathematics, Vlora, Albania

Email address: artionkashuri@gmail.com

2 Nanjing Normal University

JiANGSU Key Laboratory FOR NSLSCS,

School of Mathematical Sciences,

Nanjing Normal University, Nanjing, 210023, China

Email address: mahr.muhammad.aamir@gmail.com

3 Government College University,

Department of Mathematics,

LAhORE 54000, PAKISTAN

AND

University of Pretoria

Department of Mathematics and Applied Mathematics,

Lynnwood road, Pretoria 0002, South Africa

Email address: abbas.mujahid@gmail.com
}

Received: 13.7 .2020

Revised: 6.10 .2020

Accepted: 6.10 .2020 